\title{
Cigarette consumption and sales of nicotine replacement products
}

\author{
The-wei Hu, Hai-Yen Sung, Theodore E Keeler, Martin Marciniak
}

\begin{abstract}
Background-The first nicotine replacement product, Nicorette Gum, was marketed in 1984 as an adjuvant to help smokers quit smoking. In 1992, sales of nicotine patches were begun. Before 1996, nicotine gums and nicotine patches were prescribed by physicians and supplemented with behavioural counselling. Since 1996, nicotine gums and patches became available over the counter.

Objectives-To examine the effect of sales of nicotine replacement products on national cigarette consumption.

Design-National time series quarterly cigarette consumption, sales of nicotine gums and patches data between 1976 and 1998 are used to estimate a time series autoregressive moving average intervention model.
\end{abstract}

Participants-National reported statistics.

Main outcome measures-Per capita cigarette sales.

Results-A $10 \%$ increase in sales of nicotine replacement products will lead to a $0.04 \%$ reduction in cigarette sales. The model indicates that a $0.076 \%$ reduction in cigarette consumption is associated with the availability of nicotine patches after 1992. The over the counter dummy variable (after 1996) has a negative sign, but is not significant, perhaps due to only a few quarters of data in the study period. Conclusions-Nicotine replacement products (nicotine gums and nicotine patches) play a significant role in reducing cigarette consumption, in addition to the negative effect of increasing cigarette price and the overall trend of declining cigarette consumption (as reflected by the time trend variable). The findings of this study suggest that additional efforts for promoting sales of nicotine replacement products will be another effective alternative to discouraging cigarette consumption.

(Tobacco Control 2000;9(Suppl II):ii60-ii63)

Keywords: nicotine replacement products; nicotine gum; nicotine patches; cigarette consumption

Per capita cigarette consumption has been declining since the early 1980s, from 2757 cigarettes per year in 1982 to 1804 cigarettes in 1997 (34.6\% reduction). ${ }^{1}$ Much of the literature has attributed this decline to tobacco taxes, the dissemination of information on the negative health consequences of smoking, and the effect of health education and health promotion through means such as antismoking media campaigns and community intervention programs. However, there is a paucity of literature on the role of nicotine replacement products in the decline of cigarette consumption at the national level..$^{2-9}$

The first nicotine replacement product, Nicorette Gum, was marketed in 1984 as an adjuvant to help smokers quit smoking. Since 1992, nicotine patches and a revamped nicotine gum have been marketed. The resulting demand for these products shortly after their release was impressive, in some cases outpacing manufacturer's production capability. Before 1996, nicotine gums and nicotine patches were prescribed by physicians and supplemented with behavioural counselling as components of a smoking cessation program. In 1996, nicotine gums and patches were changed from prescription only to over the counter (non-prescription) status by the US Food and Drug Administration. This designation meant that labelling and package instructions were appropriate for safe utilisation by consumers without physician consultations. Presumably, the removal of this barrier has made these products more accessible to the general public and possibly increased the demand for them. Indeed, since the over the counter nicotine replacement products became available in the second quarter of 1996, the sales of nicotine gums and patches have almost doubled. The increasing trend in the sales of nicotine gum and patches has led to the question of what their overall importance and long term impact is on smoking cessation.

The effect of nicotine gums and patches have been examined by a few studies. The results from Pierce and colleagues, using 1993 California Tobacco Survey data, suggested that nicotine patches appear to be an important aid to smokers who want to quit. ${ }^{3}$ In particular, it aids those individuals who are using it as an adjuvant to other forms of assistance such as counselling. A study by Cromwell and colleagues examined the cost effectiveness of clinical practice for smoking cessation, including nicotine replacement therapy assisted by physicians. ${ }^{10}$ They found that it was a cost effective smoking cessation intervention. One study, conducted by Fiscella and Franks, determined the incremental cost effectiveness of the transdermal nicotine patch, indicating that the use of the nicotine transdermal patch produced one additional lifetime quitter at a cost of $\$ 7332 .^{5}$ To date, no study has examined the overall national impact of the sales of these nicotine replacement products on cigarette consumption. 


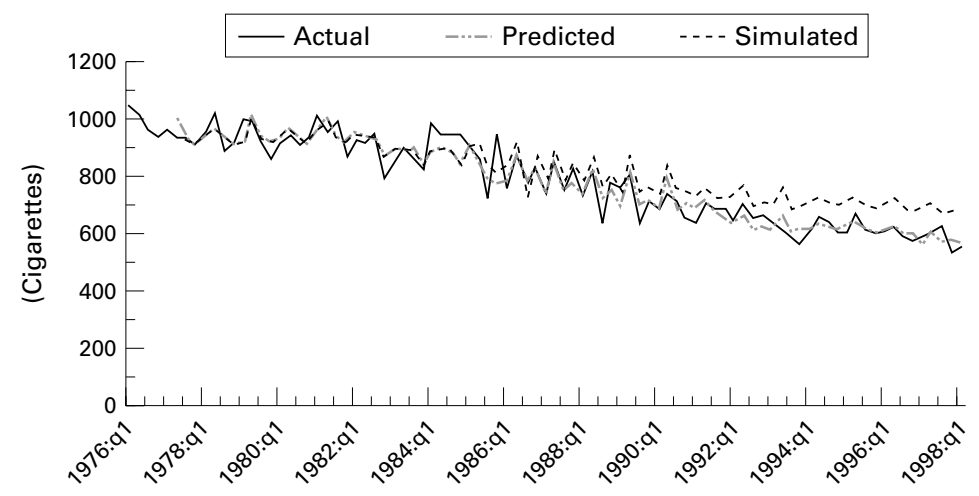

Figure 1 Per capita cigarette sales in the USA, 1976 to 1998.

The purpose of this study is to examine the effect of the sales of nicotine replacement products on national cigarette consumption in the US. This study uses the aggregate data of national cigarette consumption and sales of nicotine gums and patches. To examine the effect on per capita cigarette consumption over the past 20 years, the specified model takes into account not only the sales of nicotine replacement products, but also the price of cigarettes and the overall time trend variable. Furthermore, given the change from prescription nicotine gums and patches to over the counter sales that increased the availability to consumers, an additional dummy variable is used to examine the relative impact of the alternative means of access on cigarette consumption. The findings of this study will provide needed information on the role of nicotine replacement products in future policies for tobacco control.

\section{Methods}

DATA

Data on national cigarette consumption were obtained from the US Bureau of Alcohol, Tobacco, and Firearms, based on quantity of wholesale tax removal sales from the first quarter of 1976 through the first quarter of 1998. Cigarette consumption is expressed as the number of cigarettes sold per capita (civilian population, age 16 years and above). Figure 1 plots the quarterly per capita cigarette consumption over our study period. During the late 1970s and early 1980s, on average, adult individuals (16 years and above) consumed between 800 and 1050 cigarettes per quarter; this figure dropped to between 650 and 850 from 1987 to 1991, and has fallen further since 1993 to between 550 to 650 cigarettes per quarter.

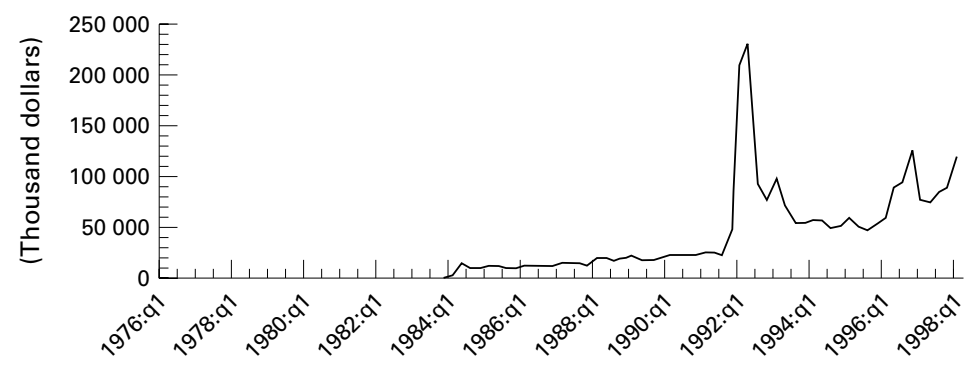

Figure 2 Total sales of nicotine gums and patches in the US, 1976 to 1998.
Data on nicotine gums and patches data are obtained from the IMS Health Incorporated MIDAS System, including all the prescription products and three major over the counter products (Nicorette Gum, Nicoderm Patch, and Nicotrol Patch.) The variable of nicotine gums and patches is expressed in wholesale value (cents) of sales per capita (civilian population, age 16 and above) in real terms (1982$84=100$ ). Because of the variation of dosage contents in different products of nicotine gums and patches, dollar value of sales should be served as a weighted amount of nicotine gums and patches consumed. Before the second quarter of 1996, these products were only available by prescription. However, since then these products have been sold over the counter. Therefore, a dummy variable is created to test the possible effect of the change in sales mode.

Figure 2 exhibits the quarterly total sales of nicotine gums and patches. The sales began at 1984 with slightly over $\$ 10$ million per quarter during 1984-87, then increased to about $\$ 24$ million in 1991. With the introduction of the nicotine patch, the dollar value of the quarterly sales jumped to over $\$ 200$ million during the first two quarters in 1992, but then declined to $\$ 90$ million beginning the third quarter of 1992 , and to between $\$ 47$ and $\$ 60$ million in 1995. However, since the availability of over the counter sales beginning in the second quarter of 1996, the total dollar sales of nicotine gums and patches almost doubled to between $\$ 75$ and $\$ 127$ million.

The cigarette price is defined as the consumer price index for tobacco products, adjusted by consumer price index for all items $(1982-84=100)$. These data are obtained from CITIBASE.

\section{STATISTICAL ANALYSIS}

The primary objective of this study is to use quarterly time series data between 1976 and 1998 to examine the effect of sales of nicotine replacement products on cigarette consumption. This is best achieved by using time series autoregressive moving average model, originally developed by Box and Jenkins ${ }^{11}$ and Box and Tiao. ${ }^{12}$ The Box and Jenkins model takes into account the seasonal variations, random fluctuations, and time trends to predict the future values of dependent variables. ${ }^{11}$ The advantage of the Box and Tiao model is its ability to incorporate additional explanatory factors, such as price, and particularly the sales of nicotine replacement products, into the model, so that effects of the sales of nicotine replacement products can be appropriately estimated. ${ }^{12}$

Thus, our specified model includes the following variables: $\mathrm{C}=$ per capita cigarette sales in logarithms; $\mathrm{Q}_{1}, \mathrm{Q}_{2}, \mathrm{Q}_{3}=$ quarterly dummy variables (fourth quarter is the comparison quarter); $\mathrm{T}=$ time trend that indicates the number of quarters from the beginning of the series with the first quarter of 1976 as $1 ; \mathrm{P}=$ real consumer price index for tobacco products $(1982-84=100)$ in logarithm; $\mathrm{N}=$ per capita wholesale sales value of nicotine gums and patches (prescription and 
over the counter) in cents in logarithm; D92 = dummy variable indicating the introduction of the sales of nicotine patches (1991 quarter $4=0.5$; after 1991 quarter 1 is 1 ; before 1991 quarter 1 is zero); D96 = dummy variable indicating the introduction of the sales of the over the counter nicotine replacement products (1996 quarter $2=0.5$; after 1996 quarter 2 is 1 ; before 1996 quarter 2 is zero); $\mathrm{L}=$ the lag operator; $\mathrm{e}=$ the random error term.

The model is as follows: $\left(1-\mathrm{C}_{1} \mathrm{~L}\right) \mathrm{C}_{\mathrm{t}}=\mathrm{a}_{0}+$ $\mathrm{a}_{1} \mathrm{Q}_{1}+\mathrm{a}_{2} \mathrm{Q}_{2}+\mathrm{a}_{3} \mathrm{Q}_{3}+\mathrm{a}_{4} \mathrm{~T}_{\mathrm{t}}+\mathrm{a}_{5} \mathrm{P}_{\mathrm{t}}+\mathrm{a}_{6} \mathrm{~N}_{\mathrm{t}-5}+$ $\mathrm{a}_{7} \mathrm{D} 92+\mathrm{a}_{8} \mathrm{D} 96+\left(1-\mathrm{b}_{1} \mathrm{~L}-\mathrm{b}_{2} \mathrm{~L}^{2}\right) \mathrm{e}_{\mathrm{t}}$

Studies of the effects of nicotine patch use on smoking cessation ${ }^{13-17}$ indicate that the use of nicotine patches has a significant effect on smoking cessation. However, it takes a longer time to achieve the intended goal. Besides, because it takes time to realise the effect of the use of nicotine gums and patches on the resulting cigarette consumption expressed by wholesale sales, a number of time periods have been tried in the empirical estimates. A lag period of five quarters has been found significant.

The above model is estimated by using the PROC ARIMA procedure with the conditional least squares method in the statistical analysis system. The method was chosen over the maximum likelihood method, because it is easier to compute the forecast values based on estimated parameters. However, the estimated parameters from both methods are very similar in our study. Various dotted lines in fig 1 demonstrate the extent to which the model fits the observed data and the simulated impact of the sales of nicotine replacement products on cigarette consumption.

\section{Results}

The estimated results presented in table 1 indicate that increasing the sales of nicotine gums and patches and the cigarette price are both significant in reducing cigarette sales in the US. The coefficients of the time trend variable indicate that cigarette consumption has been declining over time, while holding other variables in the model constant. Two dummy variables (1992 and 1996) had the expected sign, indicating a negative association between the introduction of nicotine patches or the introduction of over the counter accessibility. The coefficient of 1992 dummy variable indicates that a $0.076 \%$ reduction in cigarette consumption is associated with the availability

Table 1 Parameter estimates from quarterly time series analysis for US cigarette sales and nicotine replacement product sales (1976 to 1998, quarterly data, $n=89$ )

\begin{tabular}{lll}
\hline Variable & Coefficient & Standard error \\
\hline $\begin{array}{l}\text { Nicotine gums and } \\
\text { patches sales }\end{array}$ & $-0.004^{\star}$ & 0.002 \\
Cigarette price & $-0.260^{\star}$ & 0.114 \\
Time trend & $-0.002^{\star}$ & 0.001 \\
First quarter & 0.015 & 0.031 \\
Second quarter & $0.060^{\star \star}$ & 0.016 \\
Third quarter & 0.008 & 0.031 \\
Dummy 1992 & -0.076 & 0.030 \\
Dummy 1996 & -0.040 & 0.033 \\
Constant & $7.967^{\star \star}$ & 0.520 \\
\hline
\end{tabular}

${ }^{\star} \mathrm{p}<0.05$ by two tailed test; ${ }^{\star \star} \mathrm{p}<0.01$ by two tailed test. of nicotine patches after 1992. The coefficient is significant at the $p=0.01$ level. The over the counter dummy variable (1996) has only a few quarters of observations in the study time period (eight quarters), and perhaps does not allow enough time to evaluate the impact of the regulation. Although the variable has a negative sign, it is not significant.

Given that the magnitude of the coefficient of cigarette price variable and the sales of nicotine gums and patches variables are significant, and that both variables are expressed in logarithm, these results indicate that a $10 \%$ increase in cigarette price will lead to a reduction of almost $2.60 \%$ in cigarette sales, while a $10 \%$ increase in sales of these nicotine replacement products will lead to a $0.04 \%$ reduction in cigarette sales. It is understood that the sales value of these nicotine replacement products are relatively small when compared to the sales of cigarette products. For example, in the fiscal year 1997, the wholesale value of cigarette sales was about $\$ 30$ billion, and the sales of nicotine gums and patches were about $\$ 350$ million, about $1 \%$ of the cigarette sales. Thus the sales of nicotine patches and gum have not been a major economic threat to the tobacco industry.

An alternative method to evaluating the effect of the sales of nicotine gums and patches is to simulate and predict the sales of cigarettes as if there had been no sales of nicotine gums and patches since 1984. The predicted reduction of sales is calculated from the estimated coefficients in table 1, multiplied with the actual values of lagged five quarter nicotine gums and patches. The sale of nicotine gums began in 1984, nicotine patches in 1992, and over the counter sales of these products in 1996. From this information, the incremental effects of the sales of these products over time can be estimated. Simulated results indicate that between 1984 and 1998 , over the 15 years $(14.25$ years to be exact), a cumulative 3609 cigarettes per capita, or 63 cigarettes per capita per quarter, were not consumed as a result of the sales of nicotine gums and patches; that is equivalent to $8.3 \%$ reduction. As shown in fig 1 , the average quarterly cigarette consumption was about 700 cigarettes per capita (civilian adults, age 16 years and above) during the late 1980s; thus our estimate of the simulated effects of nicotine gums and patches on the reduction of cigarette consumption over 15 year period seems quite reasonable.

\section{Discussion}

The time series analysis, based on cigarette sales data in the US between 1976 and 1998, indicates that the sales of nicotine replacement products (nicotine gums and patches) is significant in reducing cigarette consumption, in addition to the negative effect of increasing cigarette price and the overall trend of decline (as reflected by the time trend variable). While the magnitude of the elasticity of reduction in cigarette sales with respect to sales of nicotine replacement products seems small $(0.004 \%)$ the base value of sales during 1996-98 was only about $\$ 100$ million. Thus, if there is a massive 
increase in the sales of nicotine replacement products (such as may be prompted by insurance coverage or government subsidies) from $\$ 100$ million to $\$ 150$ million, or even $\$ 200$ million, the sales of cigarettes could be reduced by $0.2 \%$ or $0.4 \%$, respectively.

There are a number of effective measures to reduce cigarette consumption, such as an increase in cigarette taxes, an increased effort in anti-smoking media campaigns, and a ban on smoking in public areas. The findings of this study suggest that additional efforts for promoting sales of nicotine gums and patches will be another effective alternative to discouraging cigarette consumption. ${ }^{18}$ Depending on relative nicotine replacement product and tobacco pricing and usage, purchasing nicotine replacement products could increase or decrease smokers' financial burden. From the consumer's point of view, there is a need to consider the cost of spending on nicotine gums or patches, the effectiveness in reducing cigarette consumption, and thus the cost savings from smoking cessation, as well as the cost savings from avoidance of incurring tobacco related illness in the long run. From the public health point of view, it would make sense for public health insurance programs as well as insurance carriers to provide coverage of prescribing nicotine gums or patches, given the cost effectiveness of using nicotine patches and gums. ${ }^{9}$ What these data show is that nicotine replacement therapy reduces tobacco consumption over time. The greater availability of these products, however, hinges on their price, access, and insurance coverage, including the availability of nicotine replacement products over the counter. ${ }^{19}$

The findings from this study are based on aggregate data. The aggregate data can provide general trends and rough estimations of the impact of sales of nicotine gums and patches. The questions of who are the users of nicotine gums and patches, how successful and relatively successful nicotine patches versus nicotine gums are, and how over the counter sales compare with physician prescription, would require additional analyses based on cross sectional data and clinical data. Furthermore, the price elasticity of the demand for nicotine gums and patches would be another piece of important information for public policy in tobacco control. These are study topics which this research team is currently pursuing.
The authors are grateful to Alison Keith and Richard Manning of Pfizer, Inc for providing data sources. These data are drawn from the IMS Health Incorporated MIDAS system; these data are proprietary to IMS Health and any analyses, opinions, and conclusions drawn from the data are solely those of the authors and do not reflect the opinion or belief of IMS Health Incorporated. This paper is prepared with funding from the Robert Wood Johnson Foundation's Substance Abuse Policy Research Program and the University of California Tobacco Related Disease Research Program. The authors alone are responsible for the content of the manuscript.

1 The Tobacco Institute. The tax burden on tobacco: historical compilation 1997, Volume 32. Washington DC: The compilation 1997, Volume

2 Kenford SL, Fiore MC, Jorenby DE, Smith SS, Wetter D, Baker TB. Predicting smoking cessation: who will quit with and without the nicotine patch. $\mathscr{F} A M A$ 1994; 271:589-94.

3 Pierce J, Gilpin E, Farkas AJ. Nicotine patch use in the general population: results from the 1993 California tobacco survey. F Natl Cancer Inst 1995;87:87-93.

4 Kornitzer M, Boutsen M, Dramaix M, Thijs J, Gustavsson $\mathrm{G}$. Combined use of nicotine patch and gum in smoking cessation: a placebo-controlled clinical trial. Prev Med 1995;24:41-7.

5 Fiscella K, Franks P. Cost-effectiveness of the transdermal nicotine patch as an adjunct to physicians' smoking cessation counseling. FAMA 1996;275:1247-51.

6 Ahuwalls J. The cost-effectiveness of the nicotine transdermal patch for smoking cessation. Prev Med 1997;26:26470.

7 Benowitz NL. Treating tobacco addiction-nicotine or no nicotine? N Engl f Med 1997;337:1230-1.

8 Lewis SF, Piasecki TM, Fiore MC, Anderson JE, Baker TB. Transdermal nicotine replacement for hospitalized patients: a randomized clinical trial. Prev Med 1998;27:296303.

9 Curry SJ, Grothaus LC, McAfee T, Pabiniak C. Use and cost effectiveness of smoking-cessation services under four insurance plans in a health maintenance organization. $N$ Engl f Med 1998;339:673-9.

10 Cromwell J, Bartosch WJ, Fiore MC, Hasselblad U, Baker T. Cost-effectiveness of the clinical practice recommendations in the AHCPR guideline for smoking cessation. Agency for Health Care Policy and Research. FAMA Agency for Health

11 Box GEP, Jenkins GM. Time series analysis: forecasting and control. Oakland, California: Holden-Day, 1976

12 Box GEP, Tiao GC. Intervention analysis applicatoins to economic and environmental problems. F Am Stat Assoc 1975;75:70-9.

13 Tonnesen P, Norregaard J, Simonsen K, Sawe U. A doubleblind trial of a 16-hour transdermal nicotine patch in smoking cessation. N Engl F Med 1991;325:311-15.

14 Daughton DM, Heatley SA, Prendergast JJ, et al. Effect of transdermal nicotine delivery as an adjunct to lowtransdermal nicotine delivery as an adjunct to low1991;151:749-52.

15 Fiore MC, Jorenby DE, Baker TB, Kenford SL. Tobacco dependence and the nicotine patch. $尹 A M A$ 1992; 268:2687-94.

16 Abelin T, Muller P, Buehler A, Vesanen K, Imhof PR. Controlled trial of transdermal nicotine patch in tobacco withdrawal. Lancet 1989;i:7-9.

17 Chu A, Zheng Y, LaVoie V, Worner TW, Li D, McCarthy E. The effects of nicotine patch use on smoking cessation. Employee Benefits fournal 1994;14:27-34.

18 Warner KE, Slade J, Sweanor DT. The emerging market for long-term nicotine maintenance. $\mathcal{F} A M A$ 1997;278:108792 .

19 Ontario Medical Association. Rethinking stop-smoking medications: myths and facts. Toronto, Ontario: Ontario Medical Association, June 1999. 